\title{
Global qualitative analysis of a quartic ecological model
}

Broer, Hendrik; Gaiko, Valery A.

Published in:

Nonlinear Analysis-Theory Methods \& Applications

DOI:

10.1016/j.na.2009.07.004

IMPORTANT NOTE: You are advised to consult the publisher's version (publisher's PDF) if you wish to cite fro it. Please check the document version below.

Document Version

Publisher's PDF, also known as Version of record

Publication date:

2010

Link to publication in University of Groningen/UMCG research database

Citation for published version (APA):

Broer, H., \& Gaiko, V. A. (2010). Global qualitative analysis of a quartic ecological model. Nonlinear Analysis-Theory Methods \& Applications, 72(2), 628-634. https://doi.org/10.1016/j.na.2009.07.004

\section{Copyright}

Other than for strictly personal use, it is not permitted to download or to forward/distribute the text or part of it without the consent of the author(s) and/or copyright holder(s), unless the work is under an open content license (like Creative Commons).

The publication may also be distributed here under the terms of Article $25 f a$ of the Dutch Copyright Act, indicated by the "Taverne" license More information can be found on the University of Groningen website: https://www.rug.nl/library/open-access/self-archiving-pure/taverneamendment.

Take-down policy

If you believe that this document breaches copyright please contact us providing details, and we will remove access to the work immediate and investigate your claim.

Downloaded from the University of Groningen/UMCG research database (Pure): http://www.rug.nl/research/portal. For technical reasons $t$ number of authors shown on this cover page is limited to 10 maximum. 


\title{
Global qualitative analysis of a quartic ecological model ${ }^{\text {t }}$
}

\author{
Henk W. Broer ${ }^{\mathrm{a}}$, Valery A. Gaiko ${ }^{\mathrm{b}, *}$ \\ a University of Groningen, Department of Mathematics, P.O. Box 407, 9700 AK Groningen, The Netherlands \\ ${ }^{\mathrm{b}}$ National Academy of Sciences of Belarus, United Institute of Informatics Problems, L.Beda Street 6-4, Minsk 220040, Belarus
}

\section{A R T I C L E I N F O}

\section{Article history:}

Received 16 February 2009

Accepted 2 July 2009

\section{MSC: \\ $34 \mathrm{C05}$ \\ $34 \mathrm{C} 07$ \\ $34 \mathrm{C} 23$ \\ $37 \mathrm{G} 05$ \\ $37 \mathrm{G} 10$ \\ 37G15}

Keywords:

Quartic ecological model

Field rotation parameter

Bifurcation

Singular point

Limit cycle

Separatrix cycle

Wintner-Perko termination principle

\begin{abstract}
A B S T R A C T
In this paper we complete the global qualitative analysis of a quartic ecological model. In particular, studying global bifurcations of singular points and limit cycles, we prove that the corresponding dynamical system has at most two limit cycles.
\end{abstract}

(C) 2009 Elsevier Ltd. All rights reserved.

\section{Introduction}

The paper is based on the applications of Bifurcation Theory and can be used for modeling problems, where system parameters play a certain role in various bifurcations. In this paper we consider a particular (quartic) family of planar vector fields, which models the dynamics of the populations of predators and their prey in a given ecological system and which is a variation on the classical Lotka-Volterra system. For the latter system the change of the prey density per unit of time per predator called the response function is proportional to the prey density. This means that there is no saturation of the predator when the amount of available prey is large. However, it is more realistic to consider a nonlinear and bounded response function, and in fact different response functions have been used in the literature to model the predator response, see $[1-10]$.

For instance, Zhu et al. [10] have studied recently the following predator-prey model:

$$
\begin{aligned}
& \dot{x}=x(a-\lambda x)-y P(x) \quad \text { (prey), } \\
& \dot{y}=-\delta y+y Q(x) \quad \text { (predator). }
\end{aligned}
$$

\footnotetext{
Acknowledgement: This work was supported by the Netherlands Organization for Scientific Research.

* Corresponding author. Tel.: +375 17 2904544; fax: +375 172842140.

E-mail address: valery.gaiko@yahoo.com (V.A. Gaiko).
} 
The variables $x>0$ and $y>0$ denote the density of the prey and predator populations respectively, while $P(x)$ is a nonmonotonic response function given by

$$
P(x)=\frac{m x}{\alpha x^{2}+\beta x+1},
$$

where $\alpha, m$ are positive and where $\beta>-2 \sqrt{\alpha}$. Observe that in the absence of predators, the number of prey increases according to a logistic growth law. The coefficient a represents the intrinsic growth rate of the prey, while $\lambda>0$ is the rate of competition or resource limitation of prey. The natural death rate of the predator is given by $\delta>0$. In Gause's model the function $Q(x)$ is given by $Q(x)=c P(x)$, where $c>0$ is the rate of conversion between prey and predator. For further discussion on the biological relevance of the model see [2-5].

In this paper we investigate the following family

$$
\begin{aligned}
& \dot{x}=x\left(1-\lambda x-\frac{y}{\alpha x^{2}+\beta x+1}\right) \quad \text { (prey), } \\
& \dot{y}=y\left(-\delta-\mu y+\frac{x}{\alpha x^{2}+\beta x+1}\right) \quad \text { (predator), }
\end{aligned}
$$

where $\alpha \geq 0, \delta>0, \lambda>0, \mu \geq 0$ and $\beta>-2 \sqrt{\alpha}$ are parameters. We note that (1.3) is obtained from (1.1) by adding the term $-\mu y^{2}$ to the second equation and after scaling $x$ and $y$, as well as the parameters and the time $t$. In this way we take into account competition between predators for resources other than prey. The non-negative coefficient $\mu$ is the rate of competition amongst predators. For examples of populations that use the group defense strategy see [1-5].

System (1.3) can be written in the form

$$
\begin{aligned}
& \dot{x}=x\left((1-\lambda x)\left(\alpha x^{2}+\beta x+1\right)-y\right) \equiv P, \\
& \dot{y}=-y\left((\delta+\mu y)\left(\alpha x^{2}+\beta x+1\right)-x\right) \equiv Q .
\end{aligned}
$$

This quartic ecological model was studied earlier, for instance, in [2-5]. However, the qualitative analysis was incomplete, since the global bifurcations of limit cycles could not be studied properly by means of the methods and techniques which were used earlier in the qualitative theory of dynamical systems.

Together with (1.4), we will also consider an auxiliary system (see [11,12])

$$
\dot{x}=P-\gamma Q, \quad \dot{y}=Q+\gamma P,
$$

applying to these systems new bifurcation methods and geometric approaches developed in [13-16] and completing the qualitative analysis of (1.4).

\section{Preliminaries}

In this paper geometric aspects of Bifurcation Theory are used and developed. First of all, the two-isocline method which was developed by Erugin is used, see [13]. An isocline portrait is the most natural construction for a polynomial equation. It is sufficient to have only two isoclines (of zero and infinity) to obtain principal information on the original polynomial system, because these two isoclines are right-hand sides of the system. Geometric properties of isoclines (conics, cubics, quartics, etc.) are well known, and all isocline portraits can be easily constructed. By means of them, all topologically different qualitative pictures of integral curves to within a number of limit cycles and distinguishing center and focus can be obtained. Thus, it is possible to carry out a rough topological classification of the phase portraits for the polynomial dynamical systems. It is the first application of Erugin's method. After studying contact and rotation properties of the isoclines, the simplest (canonical) systems containing limit cycles can be also constructed. Two groups of parameters can be distinguished in such systems: static and dynamic. Static parameters determine the behavior of phase trajectories in principle, since they control the number, position, and character of singular points in a finite part of the plane (finite singularities). The parameters from the first group determine also a possible behavior of separatrices and singular points at infinity (infinite singularities) under variation of the parameters from the second group. The dynamic parameters are field rotation parameters, see [11-13]. They do not change the number, position and index of the finite singularities, but only involve the vector field in a directional rotation. The rotation parameters allow us to control the infinite singularities, the behavior of limit cycles and separatrices. The cyclicity of singular points and separatrix cycles, the behavior of semi-stable and other multiple limit cycles are controlled by these parameters as well. Therefore, by means of the rotation parameters, it is possible to control all limit cycle bifurcations and to solve the most complicated problems of the qualitative theory of dynamical systems.

In $[13,14]$ some complete results on quadratic systems have been presented. In particular, it has been proved that for quadratic systems four is really the maximum number of limit cycles and (3:1), i.e., three limit cycles around one focus and the only limit cycle around another focus, is their only possible distribution (this is a solution of Hilbert's Sixteenth Problem in the quadratic case of polynomial dynamical systems). In [16] some preliminary results on generalizing new ideas and methods of [13] to cubic dynamical systems have already been established. In particular, a canonical cubic system of Kukles type has been constructed and the global qualitative analysis of its special case corresponding to a generalized Liénard 
equation has been carried out. It has been proved also that the foci of such a Liénard system can be at most of second order and that such system can have at most three limit cycles on the whole phase plane. Moreover, unlike all previous works on the Kukles-type systems, global bifurcations of limit and separatrix cycles using arbitrary (including as large as possible) field rotation parameters of the canonical system have been studied in [16]. As a result, the classification of all possible types of separatrix cycles for the generalized Liénard system has been obtained and all possible distributions of its limit cycles have been found. In [15] a solution of Smale's Thirteenth Problem proving that the Liénard system with a polynomial of degree $2 k+1$ can have at most $k$ limit cycles has been presented. All of these methods and results can be applied to quartic dynamical systems as well. In this paper, using [13-16], we will complete the global qualitative analysis of quartic ecological model (1.4). In particular, studying global bifurcations of singular points and limit cycles, we will prove that the corresponding dynamical system has at most two limit cycles.

\section{Singular points}

The study of singular point of system (1.4) will use two index theorems by H. Poincaré, see [11]. But first let us define the Poincaré index [11].

Definition 3.1. Let $S$ be a simple closed curve in the phase plane not passing through a singular point of the system

$$
\dot{x}=P(x, y), \quad \dot{y}=Q(x, y),
$$

where $P(x, y)$ and $Q(x, y)$ are continuous functions (for example, polynomials), and $M$ be some point on $S$. If the point $M$ goes around the curve $S$ in positive direction (counterclockwise) one time, then the vector coinciding with the direction of a tangent to the trajectory passing through the point $M$ is rotated through the angle $2 \pi j(j=0, \pm 1, \pm 2, \ldots)$. The integer $j$ is called the Poincaré index of the closed curve $S$ relative to the vector field of system (3.1) and has the expression

$$
j=\frac{1}{2 \pi} \oint_{S} \frac{P \mathrm{~d} Q-Q \mathrm{~d} P}{P^{2}+Q^{2}} .
$$

According to this definition, the index of a node or a focus, or a center is equal to +1 and the index of a saddle is -1 .

Theorem 3.1 (First Poincaré Index Theorem). If $N, N_{f}, N_{c}$, and $C$ are respectively the number of nodes, foci, centers, and saddles in a finite part of the phase plane and $N^{\prime}$ and $C^{\prime}$ are the number of nodes and saddles at infinity, then it is valid the formula

$$
N+N_{f}+N_{c}+N^{\prime}=C+C^{\prime}+1 .
$$

Theorem 3.2 (Second Poincaré Index Theorem). If all singular points are simple, then along an isocline without multiple points lying in a Poincare hemisphere which is obtained by a stereographic projection of the phase plane, the singular points are distributed so that a saddle is followed by a node or a focus, or a center and vice versa. If two points are separated by the equator of the Poincaré sphere, then a saddle will be followed by a saddle again and a node or a focus, or a center will be followed by a node or a focus, or a center.

We will use also the following theorem by A. N. Berlinskii, see [13].

Theorem 3.3 (Berlinskii Theorem). If a quadratic system (3.1) has four singular points in a finite part of the phase plane, then only one of the following cases is possible: (1) these points are vertices of a convex quadrangular, where two opposite vertices are saddles (antisaddles) and two others are antisaddles (saddles); (2) three singular points are vertices of a triangle containing the fourth point inside, and if this point is a saddle (antisaddle), then the others are antisaddles (saddles), where antisaddles are singularities that are not a saddle.

Consider system (1.4) which has two invariant straight lines: $x=0$ and $y=0$. Its finite singularities are determined by the algebraic system

$$
\begin{aligned}
& x\left((1-\lambda x)\left(\alpha x^{2}+\beta x+1\right)-y\right)=0, \\
& y\left((\delta+\mu y)\left(\alpha x^{2}+\beta x+1\right)-x\right)=0 .
\end{aligned}
$$

From (3.2), we have got: two singular points $(0,0)$ and $(0,-\delta / \mu)$, at most two points defined by the condition

$$
\alpha x^{2}+\beta x+1=0, \quad y=0,
$$

and at most four singularities defined by the system

$$
\begin{aligned}
& y=(1-\lambda x)\left(\alpha x^{2}+\beta x+1\right), \\
& y(\delta+\mu y)-x(1-\lambda x)=0,
\end{aligned}
$$

among which we always have the point $(1 / \lambda, 0)$. 
To investigate the character and distribution of the singular points in the phase plane, we will use the method developed in [13-16]. The sense of this method is to obtain the simplest (well-known) system by vanishing some parameters (usually field rotation parameters) of the original system and then to input these parameters successively one by one studying the dynamics of the singular points (both finite and infinite) in the phase plane.

Let the parameters $\alpha, \beta$ vanish and consider first the quadratic system

$$
\begin{aligned}
& \dot{x}=x(1-\lambda x-y), \\
& \dot{y}=-y(\delta+\mu y-x) .
\end{aligned}
$$

System (3.5) has four finite singularities, if $\delta \neq 1 / \lambda$. Studying isocline portraits of the equation corresponding to system (3.5) and applying Theorems 3.1-3.3, we can see that for the case, when $\delta>1 / \lambda$, system (3.5) has two saddles: $(0,0)$ and a point of intersection of two straight line-isoclines:

$$
1-\lambda x-y=0, \quad \delta+\mu y-x=0,
$$

- and two nodes: $(0,-\delta / \mu)$ and $(1 / \lambda, 0)$. For the case, when $\delta<1 / \lambda$, system $(3.5)$ has two saddles: $(0,0)$ and $(1 / \lambda, 0),-$ and two nodes: $(0,-\delta / \mu)$ and (3.6). If $\delta=1 / \lambda$, it has three singularities: a saddle $(0,0)$, a node $(0,-\delta / \mu)$, and a saddlenode $(1 / \lambda, 0)$. Since we consider the first coordinate quadrant with respect to the variables $x$ and $y$, we will be interested basically in the case of $\delta<1 / \lambda$, when the singular point defined by (3.6) is in the first quadrant.

To study singular points at infinity, consider the corresponding differential equation

$$
\frac{\mathrm{d} y}{\mathrm{~d} x}=-\frac{y(\delta+\mu y-x)}{x(1-\lambda x-y)} .
$$

Dividing the numerator and denominator of the right-hand side of (3.7) by $x^{2}(x \neq 0)$ and denoting $y / x$ by $u$, we will get the algebraic equation

$$
(1-\mu) u^{2}+(1+\lambda) u=0, \quad \text { where } u=y / x,
$$

for all infinite singularities of (3.7) except when $x=0$ (the "ends" of the $y$-axis), see [11,13]. For this special case we can divide the numerator and denominator of the right-hand side of (3.7) by $y^{2}(y \neq 0)$ denoting $x / y$ by $v$ and consider the algebraic equation

$$
(1+\lambda) v^{2}+(1-\mu) v=0, \quad \text { where } v=x / y .
$$

Eqs. (3.8) and (3.9) give three singular points at infinity for (3.7): two nodes on the "ends" of the $x$ and $y$ axes and a saddle in the direction of $u=(\lambda+1) /(\mu-1)$.

Fix the parameters $\delta, \lambda, \mu$ and take $\beta<0$ (this case will be also more interesting to us). After inputting the parameter $\beta$, we will have a cubic system:

$$
\begin{aligned}
& \dot{x}=x((1-\lambda x)(\beta x+1)-y), \\
& \dot{y}=-y((\delta+\mu y)(\beta x+1)-x) .
\end{aligned}
$$

For $\delta<1 / \lambda$ and $\beta<0$, system (3.10) has five finite singularities: two saddles $-(0,0)$ and $(1 / \lambda, 0)$, two nodes $-(0,-\delta / \mu)$ and $(-1 / \beta, 0)$, and an antisaddle (a node, a focus, or a center) defined as a point of intersection of two isoclines:

$$
\begin{aligned}
& (1-\lambda x)(\beta x+1)-y=0, \\
& (\delta+\mu y)(\beta x+1)-x=0 .
\end{aligned}
$$

For singular points at infinity, consider the corresponding differential equation

$$
\frac{\mathrm{d} y}{\mathrm{~d} x}=-\frac{y((\delta+\mu y)(\beta x+1)-x)}{x((1-\lambda x)(\beta x+1)-y)}
$$

and the algebraic equations

$$
\mu u^{2}-\lambda u=0, \quad \text { where } u=y / x,
$$

and

$$
\lambda v^{3}-\mu v^{2}=0, \quad \text { where } v=x / y,
$$

which give three infinite singularities: a node on the "ends" of the $x$-axis, a saddle-node on the "ends" of the $y$-axis, and a saddle in the direction of $u=\lambda / \mu$.

Fix the parameters $\beta, \delta, \lambda, \mu$ and take $\alpha>0$. Studying the bundle of cubic curves

$$
y=(1-\lambda x)\left(\alpha x^{2}+\beta x+1\right)
$$


which intersect at the point $(1 / \lambda, 0)$ and contact at the point $(0,1)$, we can see that system (1.4) obtained after inputting $\alpha$ has first six finite singular points: three saddles $-(0,0),(1 / \lambda, 0)$, and $\left(\left(-\beta+\sqrt{\beta^{2}-4 \alpha}\right) /(2 \alpha), 0\right)$, two nodes $-(0,-\delta / \mu)$ and $\left(\left(-\beta-\sqrt{\beta^{2}-4 \alpha}\right) /(2 \alpha), 0\right)$, and an antisaddle defined as a point of intersection of isoclines (3.4).

On increasing the parameter $\alpha$, the points $\left(\left(-\beta-\sqrt{\beta^{2}-4 \alpha}\right) /(2 \alpha), 0\right)$ and $\left(\left(-\beta+\sqrt{\beta^{2}-4 \alpha}\right) /(2 \alpha), 0\right)$ combine a saddle-node which then disappears. On further increasing $\alpha$, the point $(1 / \lambda, 0)$ becomes a triple saddle from which a saddle and a node (or a saddle-node) will appear. Thus, we will have three singular points in the first quadrant: a saddle $S$ and two antisaddles $-A_{1}$ and $A_{2}$ which are defined as points of intersection of isoclines (3.4). Suppose that with respect to the $x$-axis they have the following sequence: $A_{1}, S, A_{2}$.

To study singular points of (1.4) at infinity, consider the corresponding differential equation

$$
\frac{\mathrm{d} y}{\mathrm{~d} x}=-\frac{y\left((\delta+\mu y)\left(\alpha x^{2}+\beta x+1\right)-x\right)}{x\left((1-\lambda x)\left(\alpha x^{2}+\beta x+1\right)-y\right)}
$$

and the algebraic equations

$$
\mu u^{2}-\lambda u=0, \text { where } u=y / x,
$$

and

$$
\lambda v^{4}-\mu v^{3}=0, \text { where } v=x / y,
$$

which give three infinite singularities: a simple node on the "ends" of the $x$-axis, a triple node on the "ends" of the $y$-axis, and a simple saddle in the direction of $u=\lambda / \mu$.

Note that all results on finite singularities of system (1.4) agree with the results of [2-5,9,10], but where infinite singularities have not been investigated at all. Using the obtained information and applying the approach developed in [13-16], we can study limit cycle bifurcations of system (1.4) now. This study will use also some results obtained in $[2-5,9,10]$. In particular, the results on the cyclicity of singular points of (1.4) will be used. However, it is surely not enough to have only these results to prove the main theorem of this paper: on the maximum number of limit cycles of (1.4).

\section{Bifurcations of limit cycles}

Let us first formulate the Wintner-Perko termination principle [12] for the polynomial system

$$
\dot{\boldsymbol{x}}=\boldsymbol{f}(\boldsymbol{x}, \boldsymbol{\mu}),
$$

where $\boldsymbol{x} \in \mathbf{R}^{2} ; \boldsymbol{\mu} \in \mathbf{R}^{n} ; \boldsymbol{f} \in \mathbf{R}^{2}$ ( $\boldsymbol{f}$ is a polynomial vector function).

Theorem 4.1 (Wintner-Perko Termination Principle). Any one-parameter family of multiplicity-m limit cycles of relatively prime polynomial system $\left(4.1_{\mu}\right)$ can be extended in a unique way to a maximal one-parameter family of multiplicity-m limit cycles of $\left(4.1_{\mu}\right)$ which is either open or cyclic.

If it is open, then it terminates either as the parameter or the limit cycles become unbounded; or, the family terminates either at a singular point of $\left(4.1_{\mu}\right)$, which is typically a fine focus of multiplicity $\mathrm{m}$, or on a (compound) separatrix cycle of $\left(4.1_{\mu}\right)$, which is also typically of multiplicity $\mathrm{m}$.

The proof of this principle for general polynomial system (4.1 $1_{\mu}$ ) with a vector parameter $\boldsymbol{\mu} \in \mathbf{R}^{n}$ parallels the proof of the planar termination principle for the system

$$
\dot{x}=P(x, y, \lambda), \quad \dot{y}=Q(x, y, \lambda)
$$

with a single parameter $\lambda \in \mathbf{R}$ (see [12,13]), since there is no loss of generality in assuming that system $\left(4.1_{\mu}\right)$ is parameterized by a single parameter $\lambda$; i.e., we can assume that there exists an analytic mapping $\boldsymbol{\mu}(\lambda)$ of $\mathbf{R}$ into $\mathbf{R}^{n}$ such that $\left(4.1_{\mu}\right)$ can be written as $\left(4.1_{\mu(\lambda)}\right)$ or even $\left(4.1_{\lambda}\right)$ and then we can repeat everything, what had been done for system $\left(4.1_{\lambda}\right)$ in [12]. In particular, if $\lambda$ is a field rotation parameter of $\left(4.1_{\lambda}\right)$, the following Perko's theorem on monotonic families of limit cycles is valid (see [12]).

Theorem 4.2. If $L_{0}$ is a nonsingular multiple limit cycle of $\left(4.1_{0}\right)$, then $L_{0}$ belongs to a one-parameter family of limit cycles of $\left(4.1_{\lambda}\right)$; furthermore:

(1) if the multiplicity of $L_{0}$ is odd, then the family either expands or contracts monotonically as $\lambda$ increases through $\lambda_{0}$;

(2) if the multiplicity of $L_{0}$ is even, then $L_{0}$ bifurcates into a stable and an unstable limit cycle as $\lambda$ varies from $\lambda_{0}$ in one sense and $L_{0}$ disappears as $\lambda$ varies from $\lambda_{0}$ in the opposite sense; i.e., there is a fold bifurcation at $\lambda_{0}$. 
Applying the definition of a field rotation parameter [11-13], i.e., a parameter which rotates the field in one direction, to system (1.4), let us calculate the corresponding determinants for the parameters $\alpha$ and $\beta$, respectively:

$$
\begin{aligned}
& \Delta_{\alpha}=P Q_{\alpha}^{\prime}-Q P_{\alpha}^{\prime}=x^{3} y(y(\delta+\mu y)-x(1-\lambda x)), \\
& \Delta_{\beta}=P Q_{\beta}^{\prime}-Q P_{\beta}^{\prime}=x^{2} y(y(\delta+\mu y)-x(1-\lambda x)) .
\end{aligned}
$$

It follows from (4.2) and (4.3) that on increasing $\alpha$ or $\beta$ the vector field of (1.4) in the first quadrant is rotated in positive direction (counterclockwise) only on the outside of the ellipse

$$
y(\delta+\mu y)-x(1-\lambda x)=0 .
$$

Therefore, to study limit cycle bifurcations of system (1.4), it makes sense together with (1.4) to consider also an auxiliary $\operatorname{system~(1.5)~with~a~field~rotation~parameter~} \gamma$ :

$$
\Delta_{\gamma}=P^{2}+Q^{2} \geq 0 \text {. }
$$

Using system (1.5) and applying Perko's results, we will prove the following theorem.

Theorem 4.3. System (1.4) has at most two limit cycles.

Proof. First let us prove that system (1.4) can have at least two limit cycles. Begin with quadratic system (3.5). It is clear that such a system, with two invariant straight lines, cannot have limit cycles at all [13]. Inputting a negative parameter $\beta$ into this system, the vector field of cubic system (3.10) will be rotated in negative direction (clockwise) at infinity, the structure and the character of stability of infinite singularities will be changed, and an unstable limit, $\Gamma_{1}$, will appear immediately from infinity in this case. This cycle will surround a stable antisaddle (a node or a focus) $A_{1}$ which is in the first quadrant of system (3.10). Inputting a positive parameter $\alpha$ into system (3.10), the vector field of quartic system (1.4) will be rotated in positive direction (counterclockwise) at infinity, the structure and the character of stability of infinite singularities will be changed again, and a stable limit, $\Gamma_{2}$, surrounding $\Gamma_{1}$ will appear immediately from infinity in this case. On further increasing the parameter $\alpha$, the limit cycles $\Gamma_{1}$ and $\Gamma_{2}$ combine a semi-stable limit, $\Gamma_{12}$, which then disappears in a "trajectory concentration" [11,13].

As we saw above, on further increasing $\alpha$, two other singular points, a saddle $S$ and an antisaddle $A_{2}$, will appear in the first quadrant in system (1.4). We can fix the parameter $\alpha$, fixing simultaneously the positions of the finite singularities $A_{1}, S, A_{2}$, and consider system (1.5) with a positive parameter $\gamma$ which acts like a positive parameter $\alpha$ of system (1.4), but on the whole phase plane.

So, consider system (1.5) with a positive parameter $\gamma$. On increasing this parameter, the stable nodes $A_{1}$ and $A_{2}$ becomes first stable foci, then they change the character of their stability, becoming unstable foci. At these Andronov-Hopf bifurcations [11,13], stable limit cycles will appear from the foci $A_{1}$ and $A_{2}$. On further increasing $\gamma$, the limit cycles will expand and will disappear in small separatrix loops of the saddle $S$. If these loops are formed simultaneously, we will have a so-called eight-loop separatrix cycle. In this case, a big stable limit surrounding three singular points, $A_{1}, S$, and $A_{2}$, will appear from the eight-loop separatrix cycle after its destruction, expanding to infinity on increasing $\gamma$. If a small loop is formed earlier, for example, around the point $A_{1}\left(A_{2}\right)$, then, on increasing $\gamma$, a big loop formed by two lower (upper) adjoining separatrices of the saddle $S$ and surrounding the points $A_{1}$ and $A_{2}$ will appear. After its destruction, we will have simultaneously a big limit cycle surrounding three singular points, $A_{1}, S, A_{2}$, and a small limit cycle surrounding the point $A_{2}\left(A_{1}\right)$. Thus, we have proved that system (1.4) can have at least two limit cycles, see also $[2-5,9,10]$.

Let us prove now that this system has at most two limit cycles. The proof is carried out by contradiction applying Catastrophe Theory, see [12,13]. Consider system (1.5) with three parameters: $\alpha, \beta$, and $\gamma$ (the parameters $\delta, \lambda$, and $\mu$ can be fixed, since they do not generate limit cycles). Suppose that (1.5) has three limit cycles surrounding the only point, $A_{1}$, in the first quadrant. Then we get into some domain of the parameters $\alpha, \beta$, and $\gamma$ being restricted by definite conditions on three other parameters, $\delta, \lambda$, and $\mu$. This domain is bounded by two fold bifurcation surfaces forming a cusp bifurcation surface of multiplicity-three limit cycles in the space of the parameters $\alpha, \beta$, and $\gamma[12,13]$.

The corresponding maximal one-parameter family of multiplicity-three limit cycles cannot be cyclic, otherwise there will be at least one point corresponding to the limit cycle of multiplicity four (or even higher) in the parameter space. Extending the bifurcation curve of multiplicity-four limit cycles through this point and parameterizing the corresponding maximal one-parameter family of multiplicity-four limit cycles by the field rotation parameter, $\gamma$, according to Theorem 4.2, we will obtain two monotonic curves of multiplicity-three and one, respectively, which, by the Wintner-Perko termination principle (Theorem 4.1), terminate either at the point $A_{1}$ or on a separatrix cycle surrounding this point. Since we know at least the cyclicity of the singular point which is equal to two (see $[2-5,9,10]$ ), we have got a contradiction with the termination principle stating that the multiplicity of limit cycles cannot be higher than the multiplicity (cyclicity) of the singular point in which they terminate.

If the maximal one-parameter family of multiplicity-three limit cycles is not cyclic, using the same principle (Theorem 4.1), this again contradicts the cyclicity of $A_{1}$ (see $[2-5,9,10]$ ) not admitting the multiplicity of limit cycles to be higher than two. This contradiction completes the proof in the case of one singular point in the first quadrant.

Suppose that system (1.5) with three finite singularities, $A_{1}, S$, and $A_{2}$, has two small limit cycles around, for example, the point $A_{1}$ (the case when limit cycles surround the point $A_{2}$ is considered in a similar way). Then we get into some domain in the space of the parameters $\alpha, \beta$, and $\gamma$ which is bounded by a fold bifurcation surface of multiplicity-two limit cycles $[12,13]$. 
The corresponding maximal one-parameter family of multiplicity-two limit cycles cannot be cyclic, otherwise there will be at least one point corresponding to the limit cycle of multiplicity three (or even higher) in the parameter space. Extending the bifurcation curve of multiplicity-three limit cycles through this point and parameterizing the corresponding maximal one-parameter family of multiplicity-three limit cycles by the field rotation parameter, $\gamma$, according to Theorem 4.2 , we will obtain a monotonic curve which, by the Wintner-Perko termination principle (Theorem 4.1), terminates either at the point $A_{1}$ or on some separatrix cycle surrounding this point. Since we know at least the cyclicity of the singular point which is equal to one in this case $[2-5,9,10]$, we have got a contradiction with the termination principle (Theorem 4.1).

If the maximal one-parameter family of multiplicity-two limit cycles is not cyclic, using the same principle (Theorem 4.1), this again contradicts the cyclicity of $A_{1}$ (see $\left.[2-5,9,10]\right)$ not admitting the multiplicity of limit cycles higher than one. Moreover, it also follows from the termination principle that either an ordinary (small) separatrix loop or a big loop, or an eight-loop cannot have the multiplicity (cyclicity) higher than one in this case. Therefore, according to the same principle, there are no more than one limit cycle in the exterior domain surrounding all three finite singularities, $A_{1}, S$, and $A_{2}$.

Thus, taking into account all other possibilities for limit cycle bifurcations (see $[2-5,9,10]$ ), we conclude that system (1.4) cannot have either a multiplicity-three limit cycle or more than two limit cycles in any configuration. The theorem is proved.

\section{References}

[1] A.D. Bazykin, Nonlinear Dynamics of Interacting Populations, World Scientific, Singapore, 1998.

[2] H.W. Broer, V. Naudot, R. Roussarie, K. Saleh, Bifurcations of a predator-prey model with non-monotonic response function, C. R. Acad. Sci. Paris Ser. I 341 (2005) 601-604.

[3] H.W. Broer, V. Naudot, R. Roussarie, K. Saleh, A predator-prey model with non-monotonic response function, Regul. Chaotic. Dyn. 11 (2006) 155-165.

[4] H.W. Broer, V. Naudot, R. Roussarie, K. Saleh, Dynamics of a predator-prey model with non-monotonic response function, Discrete Contin. Dyn. Syst. Ser. A 18 (2007) 221-251.

[5] H.W. Broer, V. Naudot, R. Roussarie, K. Saleh, F.O.O. Wagener, Organizing centers in the semi-global analysis of dynamical systems, Int. J. Appl. Math. Stat. 12 (2007) 7-36.

[6] C.S. Holling, Some characteristics of simple types of predation and parasitm, Can. Entomolog. 91 (1959) 385-398.

[7] Yu.A. Kuznetsov, Elements of Applied Bifurcations Theory, Springer, New York, 2004.

[8] Y. Lamontagne, C. Coutu, C. Rousseau, Bifurcation analysis of a predator-prey system with generalized Holling type III functional response, J. Dyn. Differential Equations 20 (2008) 535-571.

[9] Y. Li, D. Xiao, Bifurcations of a predator-prey system of Holling and Leslie types, Chaos Solitons Fractals 34 (2007) 606-620.

[10] H. Zhu, S.A. Campbell, G.S.K. Wolkowicz, Bifurcation analysis of a predator-prey system with nonmonotonic functional response, SIAM J. Appl. Math. 63 (2002) 636-682.

[11] N.N. Bautin, E.A. Leontovich, Methods and ways of the qualitative analysis of dynamical systems in a plane, Nauka, Moscow, 1990 (in Russian).

[12] L. Perko, Differential Equations and Dynamical Systems, Springer, New York, 2002.

[13] V.A. Gaiko, Global Bifurcation Theory and Hilbert's Sixteenth Problem, Kluwer, Boston, 2003.

[14] V.A. Gaiko, Limit cycles of quadratic systems, Nonlinear Anal. 69 (2008) 2150-2157.

[15] V.A. Gaiko, Limit cycles of Liénard-type dynamical systems, Cubo 10 (2008) 115-132.

[16] V.A. Gaiko, W.T. van Horssen, Global bifurcations of limit and separatrix cycles in a generalized Liénard system, Nonlinear Anal. 59 (2004) 189-198. 